\title{
Neurological Rehabilitation after Severe Traumatic Brain Injury, New Tools New Hopes: The Hippotherapy Approach
}

Ana Galeote, Laure Bastien, Helene Viruega and Manuel Gaviria*

Department of Neuroscience Research, Institute Equiphoria, La Canourgue 48500, France

"Corresponding author: Manuel Gaviria, Department of Neuroscience Research, Institute Equiphoria, La Canourgue 48500 , France; Tel: +334 66 321046 ; E-mail: manuel.gaviria@equiphoria.com

Received date: Aug 20, 2014, Accepted date: Sep 06, 2014, Published date: Sep 15, 2014

Copyright: (C) 2014 Galeote A, et al. This is an open-access article distributed under the terms of the Creative Commons Attribution License, which permits unrestricted use, distribution, and reproduction in any medium, provided the original author and source are credited.

\begin{abstract}
Traumatic brain injury is an unexpected and heavily disabling event occurring mostly in young adults, frequently leading to devastating consequences for the individual and his relatives. Therapeutic alternatives are limited and neurological rehabilitation remains relatively confidential. Besides, therapeutic success when noticed is mostly empirical and need to be scientifically clarified. Hippotherapy is a rather novel therapeutic approach where by the therapist uses the horse's movement as a therapeutic intervention or support. Multimodal sensory inputs generated by the horse's rhythmic walk generate adaptive responses in the patient that conceivably promote plastic changes in brain circuits. The consolidation of motor and non-motor improvements depends partly on the congruence of the session frequency and content. In this paper, we describe the improvements noticed during a two-year' series of short programs of hippotherapy in a patient that suffered a severe traumatic brain injury in 2010 that generated permanent serious neurological and neurobehavioral sequelae.
\end{abstract}

Keywords: Traumatic brain injury; Neurological impairment; Cognitive; Sensorimotor; Brain plasticity; Hippotherapy; Neurorehabilitation.

\section{Introduction}

Worldwide, traumatic brain injury (TBI) is the leading injury cause of death and permanent disability. In the United States, there are about 1.4 million cases of TBI known per year while in France, for the same period, 155,000 cases are treated in the emergency services. Among the later, 8500 will be severely injured and will have an identified permanent disability. The high prevalence of chronic neurological and neuropsychiatric sequelae can devastate the lives of survivors and their family caregivers.

From a mechanistic point of view, there are broadly two types of forces that result in brain trauma: contact and inertial. Both of these forces are associated with two main categories of brain damage: focal damage and diffuse injury. Focal harm includes cortical and/or subcortical severe contusions and lacerations, as well as intracranial bleeding (subarachnoid hemorrhage and subdural hematoma). Diffuse injury, mainly diffuse axonal injury (DAI), is due to acceleration/ deceleration forces that lead to shearing of axons and doesn't need any skull fracture nor direct impact neither crush injury to the brain surface [1].

Brain trauma generates a complex cascade of neurochemical changes that affects brain function: deregulation of ion flux, including efflux of potassium and influx of calcium, release of excitatory neurotransmitters, mainly glutamate, further depolarization, influx of calcium ions, and widespread suppression of neurons with glucose hypometabolism. Increased activity in membrane pumps in order to restore ionic balance, raises glucose consumption, depletes energy stores, causes calcium influx into mitochondria, and impairs oxidative metabolism and consequently anaerobic glycolysis with lactate production, resulting in acidosis and edema $[2,3]$.

After TBI of any severity, a substantial amount of patients exhibits cognitive and emotional difficulties that, even without motor or sensory deficits, may prevent returning to work and other social activities. Rehabilitation after TBI is a complex process because patients' individual needs evolve with time and depend upon the severity and type of TBI, pre-morbid functional status and levels of medical and social support. Intensive intervention appears to lead to earlier gains and prompt transfer to rehabilitation services can potentially improve functional outcome and lower global hospitalization costs. However, access to neurorehabilitation services remains restricted [4].

Few laboratory studies have examined the beneficial effects of physical therapy on recovery. Emerging strategies implement specialized training protocols, such as constraint-induced movement therapy for the arm and hand [5], behavioral shaping using robotic devices [6], bilateral arm training [7], body weight-supported treadmill training [8], task oriented physical therapy [9], and music therapy [10]. Nevertheless, the reasons for the effectiveness of these treatments remain unclear. Improvement is presumably the result of synaptic changes/remodeling in response to the different inputs [11].

\section{Method}

Hippotherapy (from Greek hippos $=$ horse) is an emerging specialized rehabilitation treatment, performed on a horse under the direction of an accredited health professional (e.g. physical therapists, occupational therapists, psychomotricians, speech-language pathologists, clinical psychologists, and others). The movement of the horse at a walk is used as a therapeutic intervention or support by the therapist. During Hippotherapy, the patient has no intentional control over the horse's movement [12]. 
During Hippotherapy, the center of gravity of the horse at a walk describes a three-dimensional movement similarly to humans when walking. The adaptation of the patient to the pace rhythm is one of the key pieces of Hippotherapy. The inputs coming from the smooth and rhythmic movements made by the horse facilitate and improve patient's muscle co-contraction, joint stability, weight shift, and postural and equilibrium responses, directly targeting gross motor function [13]. The tonic rhythmic adjustment of the osteoarticular movement facilitates the transmission of a great deal of proprioceptive information. Thus the new information, determined by the horse inputs, allows the creation of new motor schemes and/or the reinforcement of existing ones [14].

A typical Hippotherapy session consists of an initial period of passive muscle relaxation and postural adjustments solely in response to the horse's movement, followed by position changes and active exercises directed by the therapist. Thus, by introducing figures such as circles or serpentines the therapist can challenge lateral weight shift and midline postural control; by lengthening the horse's stride he allows the transmission of greater movement amplitude through the patient's pelvis and trunk; by accelerating/decelerating the walk he challenges anticipatory or feedback postural control; by walking on uneven terrain he incorporates predictive visual environmental cues to the session; and so on. Moreover, the powerful thrusts of the horse's legs provide strong vestibular and proprioceptive stimulation and heighten body awareness, while repeated small postural adjustments help the patient gain a more normative sense of midline, symmetrical weight-bearing and body-image [15]. As a whole, the strong solicitation of the sensorimotor sphere promotes and interacts with mechanisms related to the executive functioning linked to the cognitive (memory, attention, executive function, speed of information processing ...), and social spheres (social comportment, motivated behavior,) [16].

\section{Case Report}

MM is a 26 years-old man who suffered a severe traumatic brain injury in September 2010 (car accident). He was initially hospitalized in an anoxic comatose state and was in a coma for 10 days. At the admission GCS scored 6 and evolved to 9-10 during the first month. The initial MRI (10/08/12) report stated important parenchymal supratentorial lesions consisting of signal abnormalities of white matter in the semioval centers of the posterior frontal region, parietal region and corpus callosum (most likely post-anoxic, non hemorrhagic), and some degree of atrophy of the hippocampus (CA, gyrusdentatus and para-hippocampalgyrus) associated with an enlargement of the temporal horns of the lateral ventricles.

Initially hospitalized in an Intensive Care Unit for two months, he was transferred to a Physical Medicine and Rehabilitation Service for eight months and was finally managed in a community Médical and Social Center for one year. As a whole, he remained hospitalized until June 2012 when he returned home. MM presents neurological signs of a frontal syndrome:

Behavioral changes and dysregulation of motor activity characterized by (i) psychomotor slowing accompanied by initiative loss in the activities of daily living (ADL), default of gesture initiative and spontaneity, deficit in the mirroring of a series of gestures and rhythms; and (ii) disorders of posture and gait consisting on occasional static and dynamic loss of balance, slow and asymmetrical locomotor movements, slow gait cadence, and permanent flexion of the hips and knees in the upright position.

Cognitive changes characterized by (i) attention and concentration deficit, short term memory deficit, absence of logic organization of data to be memorized and limitation of the field of interest; (ii) difficulty with reasoning and judging; and (iii) reduction of verbal communication associated to a lack of verbal spontaneity/initiative.

\section{Hippotherapy intervention}

The therapeutic program started in August 2012 and initially consisted of three sessions of Hippotherapy per week during three weeks. Since then, he has followed five short therapeutic programs at a rate of a session per day for one week (June 2013, August 2013, November 2013, March 2014 and July 2014).

Initially, each therapeutic program started with an evaluation session to observe the patient's current psychological state, executive coordination, walking pattern, postural control, static and dynamic balance and sensory integration. Subsequently, the therapeutic team oriented each activity towards a specific target, therefore, once the aim accomplished, the program was retuned to the next target.

A typical session started with grooming, equipment of the horse and self equipment. Then, the therapeutic team conduct the patient in riding tracks with or without reins in a rectangular 2,000 sq.m indoor arena, trick riding exercises in a round track in the indoor arena, and/or on foot workout in a $18 \mathrm{~m}$ diameter round pen. All sessions started with the horse preparation in order to enable the patient to connect with and to warm up before the session. Each exercise was designed with a specific target in mind:

Riding tracks were aiming at stimulating the cognitive functions in charge of attention, memory, anticipation, and movement initiation. Simultaneously, the horse movement provided multiple inputs that passively stimulated gait, balance, postural control and coordination. Active workout was conducted by the physiotherapist and oriented to bettering postural control, upper and lower limb coordination, static and dynamic balance, muscle reinforcement and walking pattern. Track complexity was gradually raised.

Trick riding was utilized to develop body's spatial orientation and balance reactions. The therapist progressively added complexity to the exercises in order to stimulate in the patient more accurate movements.

Round pen work focused on self-awareness and was used to observe patient's ability to check and respond to his own errors. This helped remove potential barriers to the rehabilitation.

\section{Results}

Based on the intermediate evaluations, we can state an encouraging evolution of MM's condition on different levels of the sensorimotor and cognitive spheres such as coordination and balance control, fine motor control, attention and working memory, self-initiative and selfawareness (e.g. he is capable to adjust his upper limb movements and thus, to effectively guide his horse through more complicated courses). Furthermore, we can also notice a progression on trick riding exercises: the gain in postural control benefits upper and lower limbs movement. Likewise, static and dynamic balance has gradually improved. By the end of each short therapeutic program the progression has been clear. However, a continuous improvement of 
his neurological condition has been hindered by the limited access of MM to Hippotherapy.

\section{Discussion}

As in the case of MM, subsequent long-lasting disability occurs frequently after TBI. Much of this arises from cognitive and psychomotor impairment and treatment of these drawbacks is often ineffective, but when beneficial, the mechanisms of action are frequently unclear. TBI often produces diffuse injury of white-matter connections resulting from DAI which would disconnect brain regions, and could mainly determine clinical outcome. Complex processes involve distributed neural networks and functional impairment reflect disruption of these networks. Interestingly, the network-based hypothesis emphasizes an impairment in anticipatory control which rather than associate this process to a single neural region such as the frontal lobe, consider how this aspect of brain function emerges through the interactions of several regions including frontal lobe, parietal lobe and cerebellum [17]. The anticipatory control needs both appropriate "before-the-action" motor and cognitive skills which are highly altered in the case of our patient's ADL. Interestingly, during the Hippotherapy session on the horse, the co-activation of different brain regions by means of simultaneous multimodal inputs (visual and auditory cues, sensory cues, cerebellar cues) outwardly promotes the organization of adapted responses during the exercises.

It must be emphasized that a fundamental property of the brain consists on its capacity to change in response to a broad range of inputs, including injury situations. In the latter, although there are spontaneous reparative mechanisms which follow an injury, these mechanisms are hardly ever sufficient to support substantial functional recovery even though the brain is inherently capable of changing to enable at least some behavioral restitution. The degree of plastic changes is related to both the relevance of an experience and the intensity or frequency of its constituent events [11]. According to previous works, experiences that are highly relevant are likely to produce much more rapid neuronal changes than less relevant ones. In contrast, experiences that are perceived as irrelevant may not lead to neural changes. In the same way, intensity or frequency of the experience is also crucial $[18,19]$. Since self-awareness is not deeply altered in the case of $\mathrm{MM}$, one can reasonably assume that strong psychological cues are playing a key role in making relevant the experience on the horse [20]. Indeed, the multisensory inputs that generate a balanced, stable and regular independent locomotion-like perception in the disabled patient are thought to be strong building blocks for the reconstruction of a self body-image and the idea's reinforcement of the experience's relevance.

Finally, we hypothesize that one of the main targets of the Hippotherapy approach is the cerebellum which is massively interconnected with the cerebral cortex. Conventionally, it was established that the cerebellum receives information from widespread cortical areas, including portions of the frontal, parietal, temporal, and occipital lobes. It is nowadays clear that outputs from the cerebellar nuclei project to a myriad of cortical areas, including regions of frontal, prefrontal, and posterior parietal cortex via the ventrolateral thalamus. This influences more widespread regions of the cerebral cortex than was first thought. It provides the anatomical substrate of cerebellar influence in nonmotor as well as motor areas of the cerebral cortex. Accordingly, abnormal activity in these circuits leads not only to motor deficits but also to cognitive, attentional, and affective deficits. The outputs of the cerebro-cerebellar loops provide the cerebellum with the anatomical substrate to influence the control of movement and cognition. The range of tasks associated with cerebellar activation is remarkable and includes tasks designed to assess attention, executive control, language, working memory, learning, pain, and emotion [21]. During Hippotherapy, effective use of multisensory inputs related to posture and movement would provide the basis for a reinforcement/remodeling of the cerebro-cerebellar circuits.

\section{Conclusion}

A more solid scientific groundwork for restorative neuroscience is needed. This would improve our understanding of the basis for cognitive and psychomotor integration after TBI and potentially facilitate the development of novel approaches. Understanding the effect of this injury at the level of large-scale brain networks will provide important insights for the elaboration of tailored neurorehabilitation programs. Hippotherapy represents an exciting new support both for rehabilitation of neurological disorders and for carrying on targeted research programs in neuroscience.

\section{References}

1. Blennow K, Hardy J, Zetterberg H (2012) The neuropathology and neurobiology of traumatic brain injury. See comment in PubMed Commons below Neuron 76: 886-899.

2. Giza CC, Hovda DA (2001) The Neurometabolic Cascade of Concussion. See comment in PubMed Commons below J Athl Train 36: 228-235.

3. Barkhoudarian G, Hovda DA, Giza CC (2011) The molecular pathophysiology of concussive brain injury. See comment in PubMed Commons below Clin Sports Med 30: 33-48, vii-iii.

4. Kolias AG, Guilfoyle MR, Helmy A, Allanson J, Hutchinson PJ (2013) Traumatic brain injury in adults. See comment in PubMed Commons below PractNeurol 13: 228-235.

5. Wolf SL, Blanton S, Baer H, Breshears J, Butler AJ (2002) Repetitive task practice: a critical review of constraint-induced movement therapy in stroke. See comment in PubMed Commons below Neurologist 8: 325-338.

6. Hidler J, Nichols D, Pelliccio M, Brady K, Campbell DD, et al. (2009) Multicenter randomized clinical trial evaluating the effectiveness of the Lokomat in subacute stroke. See comment in PubMed Commons below Neurorehabil Neural Repair 23: 5-13.

7. Lin KC, Chen YA, Chen CL, Wu CY, Chang YF (2010) The effects of bilateral arm training on motor control and functional performance in chronic stroke: a randomized controlled study. See comment in PubMed Commons below Neurorehabil Neural Repair 24: 42-51.

8. Duncan PW, Sullivan KJ, Behrman AL, Azen SP, Wu SS, et al. (2007) Protocol for the Locomotor Experience Applied Post-stroke (LEAPS) trial: a randomized controlled trial. See comment in PubMed Commons below BMC Neurol 7: 39.

9. Jonsdottir J, Cattaneo D, Recalcati M, Regola A, Rabuffetti M, et al, (2010) Task-oriented biofeedback to improve gait in individuals with chronic stroke: motor learning approach. See comment in PubMed Commons below Neurorehabil Neural Repair 24: 478-485.

10. Schneider S, Schönle PW, Altenmüller E, Münte TF (2007) Using musical instruments to improve motor skill recovery following a stroke. See comment in PubMed Commons below J Neurol 254: 1339-1346.

11. Kolb B, Muhammad A (2014) Harnessing the power of neuroplasticity for intervention. See comment in PubMed Commons below Front Hum Neurosci 8: 377.

12. Sterba JA (2007) Does horseback riding therapy or therapist-directed Hippotherapy rehabilitate children with cerebral palsy? See comment in PubMed Commons below Dev Med Child Neurol 49: 68-73. 
Citation: Galeote A, Bastien L, Viruega H, Gaviria M (2014) Neurological Rehabilitation after Severe Traumatic Brain Injury, New Tools New Hopes: The Hippotherapy Approach. J Neurol Neurophysiol 5: 231. doi:10.4172/2155-9562.1000231

Page 4 of 4

13. Sterba JA, Rogers BT, France AP, Vokes DA (2002) Horseback riding in children with cerebral palsy: effect on gross motor function. See comment in PubMed Commons below Dev Med Child Neurol 44: 301-308.

14. Debuse D, Chandler C, Gibb C (2005) An exploration of German and British physiotherapists' views on the effects of Hippotherapy and their measurement. See comment in PubMed Commons below Physiother Theory Pract 21: 219-242.

15. McGibbon NH, Benda W, Duncan BR, Silkwood-Sherer D (2009) Immediate and long-term effects of Hippotherapy on symmetry of adductor muscle activity and functional ability in children with spastic cerebral palsy. Arch Phys Med Rehabil 90: 966-974.

16. Koziol LF, Lutz JT (2013) From movement to thought: the development of executive function. See comment in PubMed Commons below ApplNeuropsychol Child 2: 104-115.
17. Ham TE, Sharp DJ (2012) How can investigation of network function inform rehabilitation after traumatic brain injury? See comment in PubMed Commons below CurrOpinNeurol 25: 662-669.

18. Diaz Heijtz R, Kolb B, Forssberg H (2003) Can a therapeutic dose of amphetamine during pre-adolescence modify the pattern of synaptic organization in the brain? See comment in PubMed Commons below Eur J Neurosci 18: 3394-3399.

19. Horn G (2004) Pathways of the past: the imprint of memory. See comment in PubMed Commons below Nat Rev Neurosci 5: 108-120.

20. Ham TE, Bonnelle V, Hellyer P, Jilka S, Robertson IH, et al. (2014) The neural basis of impaired self-awareness after traumatic brain injury. See comment in PubMed Commons below Brain 137: 586-597.

21. Strick PL, Dum RP, Fiez JA (2009) Cerebellum and nonmotor function. See comment in PubMed Commons below Annu Rev Neurosci 32: 413-434. 